\title{
A Simple Strategy for Capturing the Unstable Steady Solution of an Unsteady Flow
}

\author{
Weiwei Zhang*, Yilang Liu, Jing Li \\ College of Aeronautics, Northwestern Polytechnical University, Xi'an, China \\ Email: ${ }^{*}$ aeroelastic@nwpu.edu.cn
}

Received 8 May 2015; accepted 21 June 2015; published 24 June 2015

Copyright (C) 2015 by authors and Scientific Research Publishing Inc.

This work is licensed under the Creative Commons Attribution International License (CC BY). http://creativecommons.org/licenses/by/4.0/

cc) (i) Open Access

\begin{abstract}
A simple strategy for capturing unstable steady solution is proposed in this paper. By enlarging the real time step, the unstable high frequency modes of the unsteady flow can be effectively suppressed because of the damping action on the real time level. The steady component will not be changed during real time marching. When the unsteady flow solution converges to a steady state, the partial derivatives of real time in unsteady governing equations will disappear automatically. The steady solution is the exacted unstable steady solution. The method is validated by the laminar flow past a circular cylinder and a transonic buffet of NACA0012 airfoil. This approach can acquire high-precision unstable steady solutions quickly and effectively without reducing the spatial discretization accuracy. This work provides the basis for unstable flow modeling and analysis.
\end{abstract}

\section{Keywords}

\section{Unstable Steady Solution, Real Time Step, Modeling, Unsteady Flows}

\section{Introduction}

Computational fluid dynamics (CFD) has been developed for over 30 years and is able to intensively simulate the complex flow and unstable process of temporal evolution. However, in many engineering studies, particularly for multidisciplinary analysis such as fluid-structure interaction and flow control issues, the direct CFD method is time consuming and difficult to implement for qualitative analysis and parameterization design. Thus, in the last 10 years, reduced order methods [1]-[3] based on the unsteady flow field have been developed rapidly and have been widely used in various engineering fields [4]-[8].

Most reduced order methods are executed on the basis of external excitation for the response to a steady state. These methods are still linear models, which are convenient for stability analysis and control law designing.

"Corresponding author. 
Modelling and reducing orders for the unsteady flow-field perturbations based on a steady-state solution are the essence of the linear method. Therefore, obtaining the steady-state numerical solution is the fundamental job of this method. Obtaining the steady-state solution is simple for stable flows. By contrast, acquiring the steadystate solution is usually difficult for unstable flows. However, most problems involved in engineering are closely related to unstable flows, such as vortex-induced vibration problems [9] [10] and buffet problems in aeronautics [11]-[15]. Analyzing the flow physical mechanisms and flow-field stability control is significant to model unstable flows.

Unstable flows can be modelled in two ways. One approach involves linearizing the control equations on the basis of steady-state solutions and obtaining the linear equations of small disturbance unsteady components for further reduction [14]-[16]. The other approach uses closed-loop control to transform the unstable flow to stable flow, creates a random perturbation for the stable flow, and institutes dynamic models by the system identification method [17] [18]. The key point of the second approach is that the designed actuating pattern and control law can turn the unstable flow to a stable flow. Designing the control laws of a complex, nonlinear, and unsteady flow is also difficult. The foundation of the first modelling approach is obtaining the steady-state solution of unstable flows, which usually involves two methods. The first method imposes forced symmetry boundary conditions [19]. However, this method is only suitable for symmetric problems, such as flows past a circular cylinder. This method is invalid for asymmetric problems, such as dislocation tandem cylinders, airfoil at high angles of attack with dynamic separation, and transonic buffet problems. The second method needs a feedback control process to obtain a steady solution [17] [20]. Designing an appropriate actuating pattern and control law to keep the flow stable is also difficult. A few literature works proposed the use of the time mean flow solution instead of the unstable steady solution. However, the topology of both solutions is significantly different from each other. Moreover, the characteristics of disturbance solutions are observed [19] [21]-[23]. Other literature works have adopted the subcritical flow before instability to substitute for the unstable steady solution [24] [25]. This method is simple to implement but only takes an expectant effect in the vicinity of an instability critical point; away from the critical point, the method will produce significant errors.

This paper proposes a simple strategy for capturing an unstable steady solution. For the implicit dual timestepping scheme, the high frequency unstable modes will be filtered by enlarging the real time step substantially such that only the steady component remains. Thus, we can acquire a high-precision steady solution that satisfies the control equations and boundary conditions. The effectiveness of the method is verified by a typical flow-field solution including the flow past a circular cylinder and the transonic buffet problem of NACA0012 airfoil. Furthermore, we also analyze the mechanism of the impact on the real time step for the unstable flows.

\section{Numerical Method}

\subsection{Governing Equations and Finite-Volume Formulation}

The 2D Navier-Stokes flow equations can be written in integral form as follows:

$$
\frac{\partial}{\partial t} \iint_{\Omega} \boldsymbol{Q} \mathrm{d} \Omega+\oint_{\partial \Omega} \boldsymbol{F}(\boldsymbol{Q}) \cdot \boldsymbol{n} \mathrm{d} \Gamma=\oint_{\partial \Omega} \boldsymbol{G}(\boldsymbol{Q}) \cdot \boldsymbol{n} \mathrm{d} \Gamma
$$

where $\Omega$ is the control volume, $\partial \Omega$ is the boundary of the control volume, and $\boldsymbol{n}=\left(n_{x}, n_{y}\right)^{\mathrm{T}}$ is the outer normal vector of the control volume boundary. The vector $\boldsymbol{Q}$ are conservative variables, $\boldsymbol{F}(\boldsymbol{Q})$ and $\boldsymbol{G}(\boldsymbol{Q})$ denote inviscid fluxes and viscous fluxes respectively.

In the cell-centered finite-volume method, the computational domain is divided into non-overlapping control volumes that completely cover the domain. The interface variables are derived from the average values of the grid cells to calculate the flux of control volumes. The equations of the integral form are translated to linear ordinary differential equations through spatial discretization, and the flow variables are obtained by the timemarching method.

For the second-order finite-volume method, the semi-discrete finite-volume formulation of the flow equations is expressed as follows:

$$
\frac{\mathrm{d} \boldsymbol{Q}_{i}}{\mathrm{~d} t}=-\frac{1}{\left|\Omega_{i}\right|} \sum_{m=1}^{N(i)}\left|\Gamma_{i, m}\right|\left(\boldsymbol{F}\left(\boldsymbol{Q}_{i, m}\right)-\boldsymbol{G}\left(\boldsymbol{Q}_{i, m}\right)\right) \cdot \boldsymbol{n}_{i, m}
$$

where $\boldsymbol{Q}_{i}$ denotes the cell-centered value of the control volume $i, N(i)$ is the sum of cell faces and $\Gamma_{i, m}$ is 
the interface area.

The numerical flux can be evaluated by the upwind scheme. According to the Godunov-type method, the interface normal flux is calculated by the Riemann flux:

$$
\boldsymbol{F}\left(\boldsymbol{Q}_{i, m}\right) \cdot \boldsymbol{n}_{i, m} \approx \boldsymbol{F}\left(\boldsymbol{Q}_{i, m}^{L}, \boldsymbol{Q}_{i, m}^{R}, \boldsymbol{n}_{i, m}\right)
$$

In the current paper, we mainly adopt the Roe scheme [26] to evaluate the numerical flux. For the secondorder accuracy method, the middle point of the control volume interface is used to calculate the numerical integral.

\subsection{Conditions for Acquiring Unstable Steady Solution by Enlarging the Real Time Step}

\subsubsection{Dual Time-Stepping for Implicit Scheme}

For the semi-discrete Equation (2), we adopt the implicit discretization for flux terms and the three-point backward-difference approximation of the time derivative:

$$
\frac{3 \boldsymbol{Q}_{i}^{n+1}-4 \boldsymbol{Q}_{i}^{n}+\boldsymbol{Q}_{i}^{n-1}}{2 \Delta t}+\boldsymbol{R}_{i}\left(\boldsymbol{Q}_{i}^{n+1}\right)=0
$$

where

$$
\boldsymbol{R}_{i}\left(\boldsymbol{Q}_{i}^{n+1}\right)=\frac{1}{\left|\Omega_{i}\right|} \sum_{m=1}^{N(i)}\left|\Gamma_{i, m}\right|\left(\boldsymbol{F}\left(\boldsymbol{Q}_{i, m}\right)-\boldsymbol{G}\left(\boldsymbol{Q}_{i, m}\right)\right) \cdot \boldsymbol{n}_{i, m}
$$

The symbol $\Delta t$ denotes the real time step, and the superscript $n+1$ is the value of the new time level. Equation (4) is solved by the implicit dual time-stepping scheme, which is a particular technique often employed for unsteady flows proposed by Jameson [27]. The main idea of the dual time-stepping approach is adding the pseudo-time level to the left of the governing equations. Thus, we obtain the following:

$$
\frac{\mathrm{d} \boldsymbol{Q}_{i}^{n+1}}{\mathrm{~d} \tau}+\frac{3 \boldsymbol{Q}_{i}^{n+1}-4 \boldsymbol{Q}_{i}^{n}+\boldsymbol{Q}_{i}^{n-1}}{2 \Delta t}+\boldsymbol{R}_{i}\left(\boldsymbol{Q}_{i}^{n+1}\right)=0
$$

where $\tau$ denotes the pseudo-time variable. The implicit time-marching scheme can be employed for the solution of the system of Equation (6) on the pseudo-time level. When the scheme is fulfilled, i.e., $\mathrm{d} \boldsymbol{Q}_{i}^{n+1} / \mathrm{d} \tau=0$, we obtain a steady state in pseudo time. The real solution of the new time level $\boldsymbol{Q}_{i}^{n+1}$ is also obtained. The significant advantage of this approach is that the physical time step is unrestricted as in explicit methods.

\subsubsection{Mechanism Analysis of the Impact on Real Time Step for the Response of the Unsteady Flow}

For the unsteady flow, the unsteady effect in numerical method is mainly reflected by the real time derivative in the governing equations. However, the explicit time-marching scheme must adopt a small time step to ensure the numerical stability because of the restriction of the CFL condition. Thus, the calculation efficiency is low. Therefore, the implicit time-marching scheme is generally used in the unsteady flow-field solution. The process for solving numerical governing equations includes decomposing the computational domain with small nonoverlapping control volumes and transforming the continuous partial differential equations into discrete linear algebraic equations to solve. Considering the truncation error caused by the equation approximation and the dissipation introduced by the numerical scheme, the numerical solution cannot completely and exactly describe the real flow field. Hence, we acquired an approximate simulation. With regard to the physical unsteady flow, such as periodic flow, vortex shedding, and high angle of attack separation flow, the strength of its unsteady characteristics is mainly determined by the instability of flow itself. The necessary condition for the numerical method to accurately simulate the unsteady flow is that the dissipation introduced by the method must be less than the flow instability characteristics. A higher numerical method accuracy corresponds to a smaller solution dissipation.

In general, the steady solution of the unstable flow cannot be obtained by solving the steady governing equations. However, given the dissipation action of the numerical scheme, which embodies the damping effect in flow-field solving, solving the steady governing equations directly can still acquire the steady-state solution if the numerical damping is greater than the flow instability for a few weak unsteady flows. However, for a strong unsteady flow, numerical damping can insufficiently suppress the flow instability, particularly the high-preci- 
sion method with low dissipation. Furthermore, the steady-state solution cannot be obtained naturally. Thus, many studies adopt coercive boundary conditions to achieve this solution. Expanding the scale of computational grids or using low-order accuracy spatial discrete schemes is the most straightforward way to increase the numerical dissipation. Although this manner may also acquire the steady-state solution, such an approach will greatly reduce the numerical solution accuracy and result in incorrect computation.

We can quickly, efficiently, and accurately obtain the unstable steady solution by enlarging the real time step for unsteady flow-field equations. For the unsteady flows, the flow field contains different magnitude of frequency modes. We need to adopt very small real time step to exactly capture the high frequency modes. While, when Enlarged the real time step, the high frequency modes of unsteady flow are filtered out, whereas the low frequency stable mode will not change. Therefore, enlarging the real time step is equivalent to increasing the numerical dissipation of the real time level. With the proceeding of time marching, when all of the unstable frequency modes decay and only the stable frequency modes remain, the flow field tends to a steady state. In Equation (10), when iterations in the pseudo time-level converge, the pseudo-time derivative $\mathrm{d} \boldsymbol{Q}_{i}^{n+1} / \mathrm{d} \tau=0$ tends to be zero and the real solution in the new time level is obtained. When all of the unstable modes are filtered out, the real time derivative $\left(3 \boldsymbol{Q}_{i}^{n+1}-4 \boldsymbol{Q}_{i}^{n}+\boldsymbol{Q}_{i}^{n-1}\right) / 2 \Delta t$ also tends to be zero, and the unstable steady solution is obtained. Furthermore, the steady solution satisfies the steady flow control equations and boundary conditions and does not reduce the numerical accuracy. Thus, we obtain the steady solution for the unstable flow field.

\section{Numerical Examples}

\subsection{Laminar Flow past a Circular Cylinder}

Laminar flow past a stationary circular cylinder is one of the most classical subjects of fluid mechanics and is the fundamental model of investigating the complex flow. Modeling and analyzing the flow past a cylinder can also deepen the understanding of flow physical mechanisms. This section mainly analyzes two states of the Reynolds number including $\mathrm{Re}=60$ and $\mathrm{Re}=100$.

In this case, the laminar flow past a stationary cylinder has a free stream of $M_{\infty}=0.1$ and the Reynolds number is 60 and 100 . The computational domain is divided by 40324 control volumes and 200 points on the cylinder surface. Furthermore, a hybrid mesh is adopted wherein the first height of the boundary layer is $5 \times$ $10^{-3}$, the layer sum is 11 , and the total height is 0.1 . The meshes of the computational domain and the grids near the cylinder are shown in Figure 1. The Roe flux scheme and implicit dual-time marching method are chosen to calculate, and the pseudo-time iteration method is symmetric Gauss-Seidel with a residual error convergence level $5 \times 10^{-8}$.

For the two different states, the Stouhal numbers of the shedding vortex are $S t_{\mathrm{Re}=60}=0.135$ and $S t_{\mathrm{Re}=100}=$ 0.161 and correspond to the periods $T_{\mathrm{Re}=60}=74.1$ and $T_{\mathrm{Re}=100}=62.5$, which are uniformed by the non-dimensional parameter of the diameter of the cylinder and free-stream sound speed, respectively. First, we set the real time step as $d$ treal $=0.01 T$, i.e., 100 points are computed in a single period. The results are shown in Figure 2 . The vorticity of the flow field behind the cylinder is shown in Figure 2(a), and the lift coefficient response of the cylinder is shown in Figure 2(b). The pressure distribution and streamlines of the time mean flow solution at this state are shown in Figure 3. However, when we enlarge the real time step to $d t r e a l=T$, a couple of symmetric standing vortex is behind the cylinder and the flow field maintains stability at all times. The results are
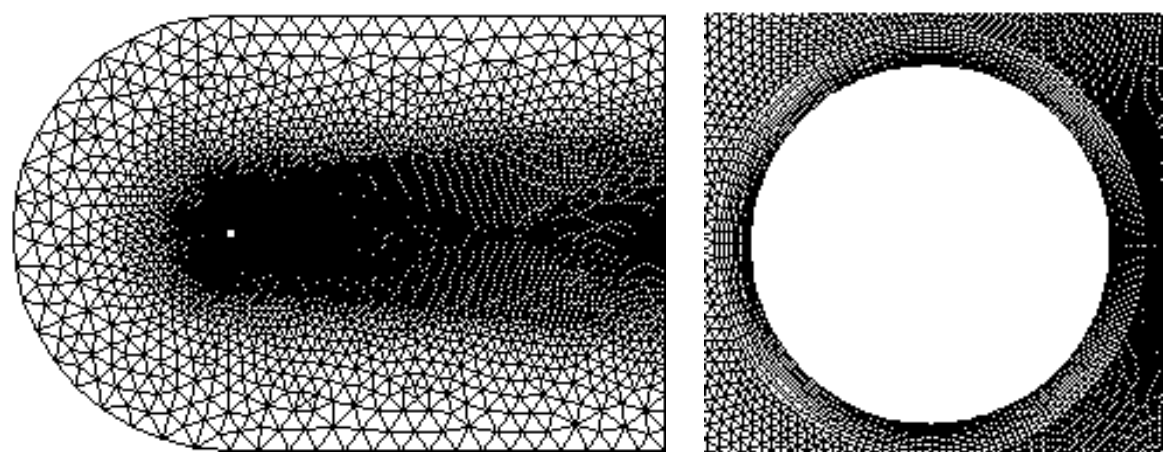

Figure 1. Meshes of the circular cylinder. 

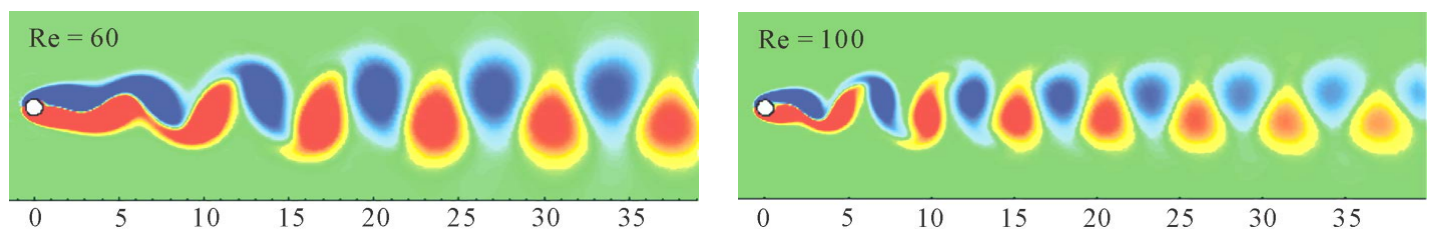

(a)
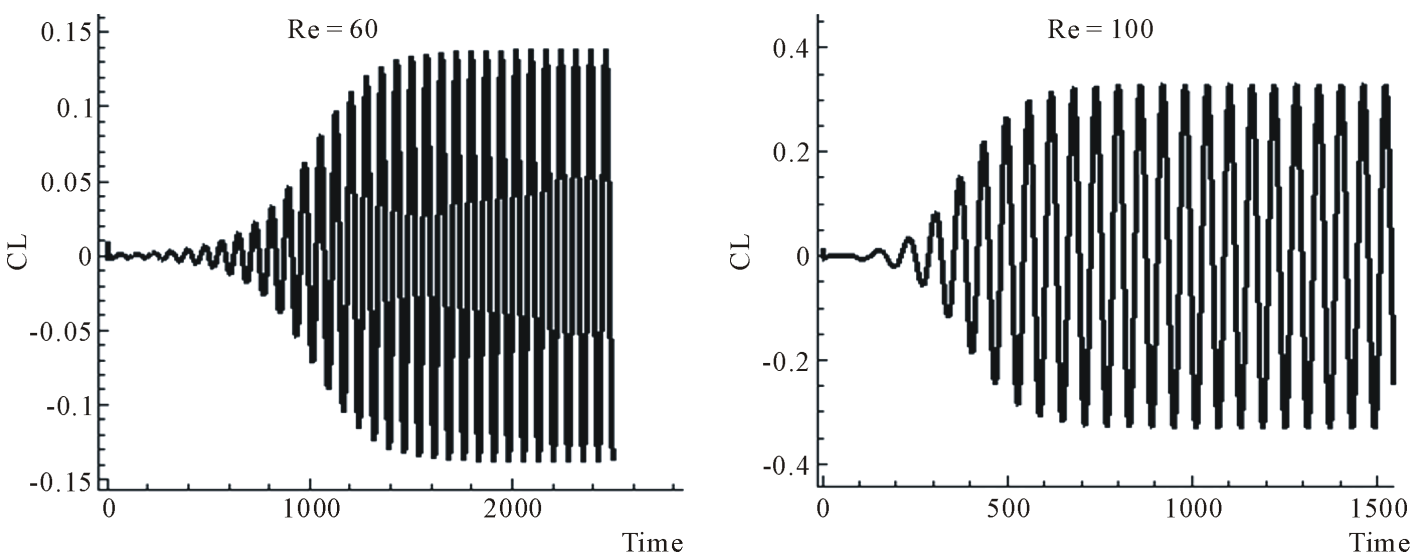

(b)

Figure 2. Results of the circular cylinder for the real time step dtreal $=T$. (a) Vorticity of flow field behind the cylinder; (b) Lift coefficient response of the cylinder.

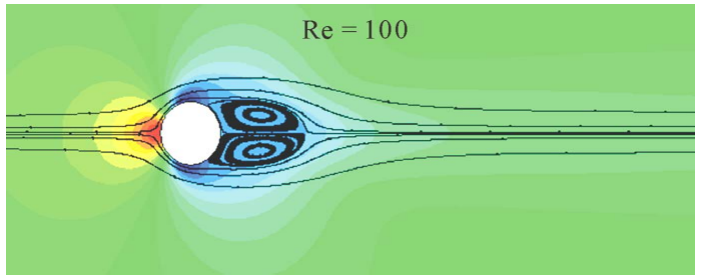

Figure 3. Pressure distribution and streamlines of the time mean flow with $\mathrm{Re}=100$.

shown in Figure 4. The streamlines of the flow field are shown in Figure 4(a), the vorticity comparisons of time-averaged flow and unstable steady flow with referenced paper [23] are shown in Figure 4(b). The lift coefficient response of the cylinder is displayed in Figure 4(c).

From the above results, when the real time step is small, the unsteady flow field can be accurately simulated by the numerical method. The shedding vortex behind the cylinder is significantly captured and the lift coefficient results are calculated accurately. When increasing the real time step to the vortex shedding period, the unstable flow field will transform to the steady state and do not change with time. The lift coefficient curve also decreases rapidly to nearly zero. The vorticity distribution of both the time-mean flow and unstable steady flow are all very close to the referenced article (Figure 4(b)). Therefore, the steady flow field obtained by this method does not reduce the accuracy and has high precision.

We perform a calculation by using different sizes of real time step and depict the change of lift coefficient amplitude in Figure 5. In this figure, the $y$-axis denotes the amplitude of the lift coefficient and the $x$-axis denotes the multiple of the shedding vortex period $T$. For the weak unstable flow, $\operatorname{Re}=60$, the flow field will tend to show a steady state when the real time step increases to $4 \% T$. For the strong unstable flow, i.e., $R e=100$, the flow field will tend to show a steady state when the real time step increases to $T / 2$. Thus, the critical real time step of acquiring the steady flow field is related to the instability strength of the flow field. A stronger instability characteristic corresponds to a larger critical real step size.

After obtaining the unstable steady solution, we can recalculate the real unsteady flow field on the basis of the solution as the initial flow field. The lift coefficient response of the cylinder with $\mathrm{Re}=100$ and $d$ treal $=0.01 T$ is 

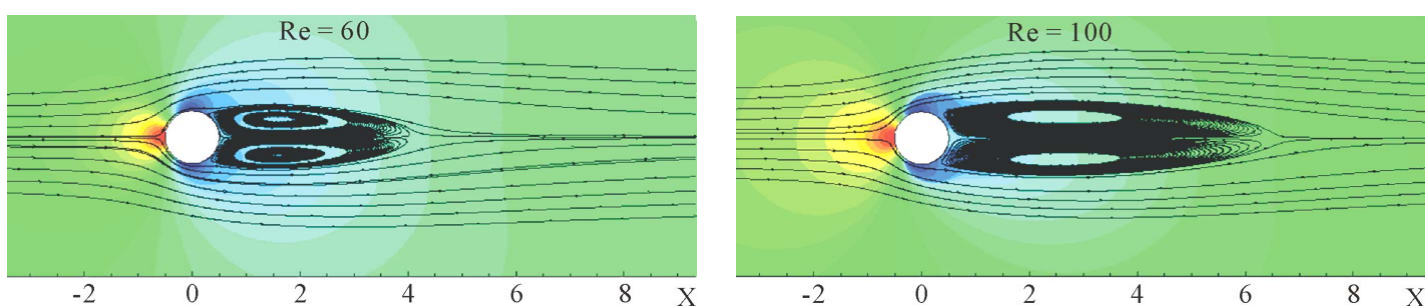

(a)

Steady

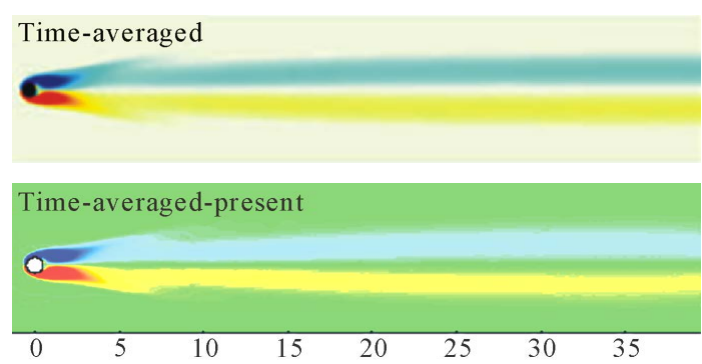

(b)
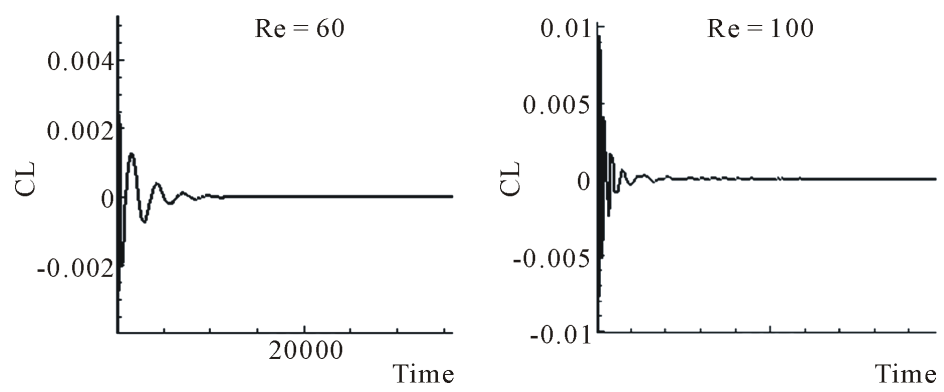

(c)

Figure 4. Results of the circular cylinder for the real time step $d$ treal $=T$. (a) Pressure distribution and streamlines of the flow field; (b) Vorticity comparisons of the unstable steady flow and time-averaged flow: the upper results are obtained from the referenced paper [23], and the lower results are obtained by the present method; (c) Lift coefficients response of the cylinder.
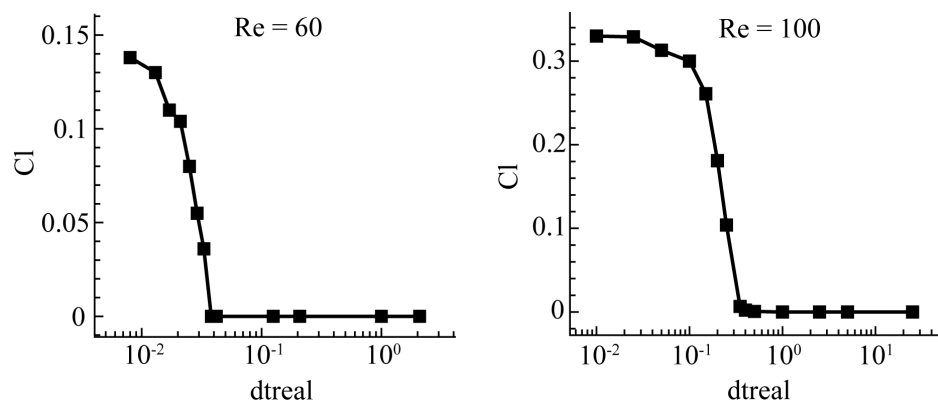

Figure 5. Change of lift coefficient amplitude with different sizes of real time step.

shown in Figure 6. A comparison of the results shown in Figure 2(b) shows that a slow and detailed process can be caught from the stable state to the unsteady flow field. By using the unstable steady solution, we can make a better analysis of period flow.

\subsection{Transonic Buffet Flow of NACA0012 Airfoil}

Transonic buffet is a phenomenon of forced vibration because of the interaction between shock waves and 
boundary layer. This phenomenon is a technical challenge that is often encountered in aircraft design. Modeling and analyzing the buffet flow are particularly important to investigate the mechanism and mitigate or control the buffet vibration. Obtaining the steady-state solution of the unstable flow is the fundamental work of this method. This section mainly demonstrates the transonic buffet flow as the case of NACA0012 airfoil.

In this case, the flow past a NACA0012 airfoil has an angle of attack of $\alpha=5.5^{\circ}$ and the free stream $M_{\infty}=0.7$ and Reynolds number is $3.0 \times 10^{6}$. The computational domain is divided by 8568 control volumes and 180 points on the airfoil surface. A hybrid mesh is adopted wherein the first height of the boundary layer is $1 \times 10^{-5}$ and the total height is 0.02 . The meshes of the computational domain and grids near the cylinder are shown in Figure 7. We adopt the SA turbulence model, and other computational method settings are similar to those in Section 3.1.

Given that the reduced frequency of this state is $k=0.195$, the corresponding flow period is $T=23$, which is uniformed by the non-dimensional parameter of the chord length of the airfoil and the free-stream sound speed. First, we set the real time step as $d t r e a l=0.01, T=0.23$, i.e., 100 points are computed in a single period. The results are shown in Figure 8. The pressure contours and streamlines of four different times in a period are shown in Figure 8(a), and the lift coefficient response of the airfoil is shown in Figure 8(b). The pressure distribution and streamlines of the time mean flow solution at this state are shown in Figure 9. However, when we enlarge the real time step to $d t r e a l=T$, a stable state flow field is obtained and the lift coefficient converges. The results are shown in Figure 10. The pressure contours and streamlines are shown in Figure 10(a), and the lift coefficient response of the airfoil is shown in Figure 10(b). The comparison of pressure coefficients with time-mean flow and unstable steady flow is shown in Figure 10(c).

From the above results, when the real time step is equal to $0.01 T$, the flow field quickly develops to the unstable state and a buffet phenomenon significantly occurs. When the real time step increases to $d t r e a l=T$, the flow field no longer presents an unsteady state. The vortex is stationary on the upper airfoil, and the lift coefficient converges and does not change with time. By comparing pressure contours and pressure coefficients, significant

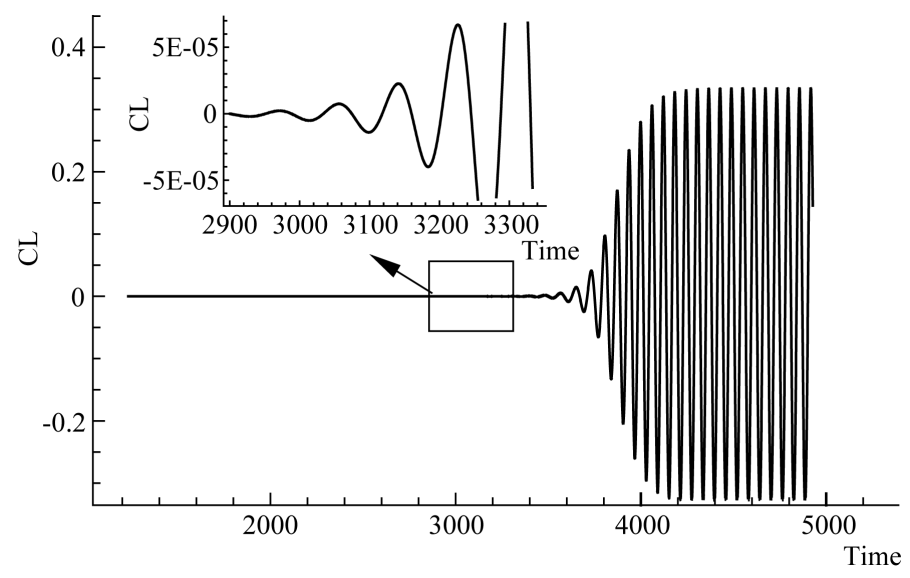

Figure 6. Lift coefficient response of the cylinder based on the steady solution for $\operatorname{Re}=100$.
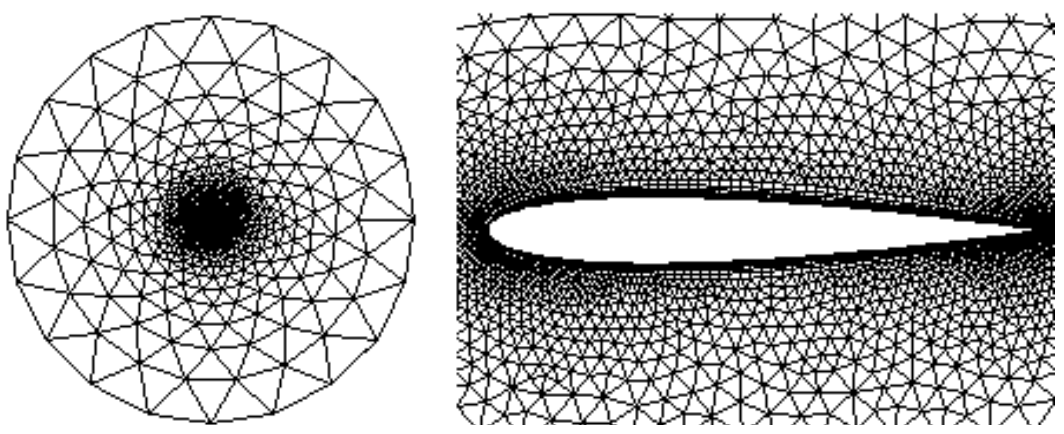

Figure 7. Computational domain grids of NACA0012 airfoil. 

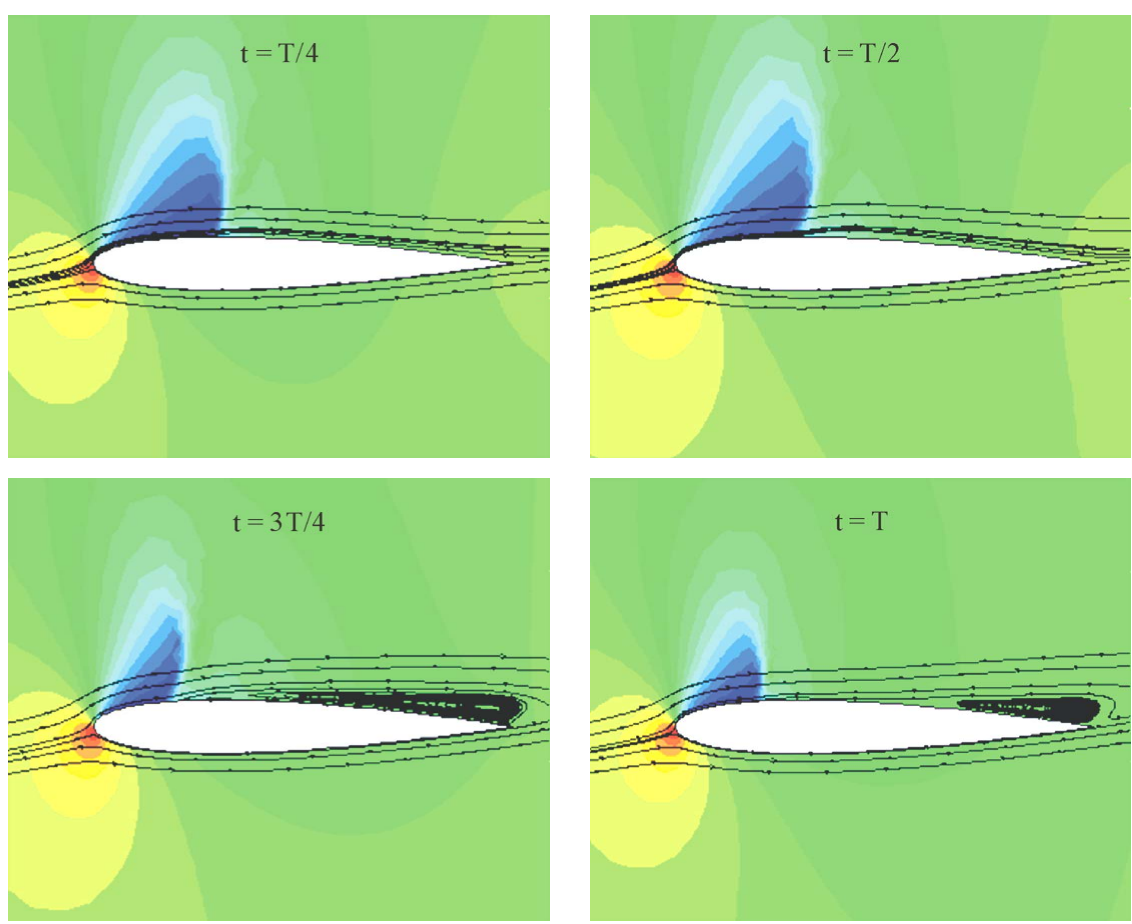

(a)

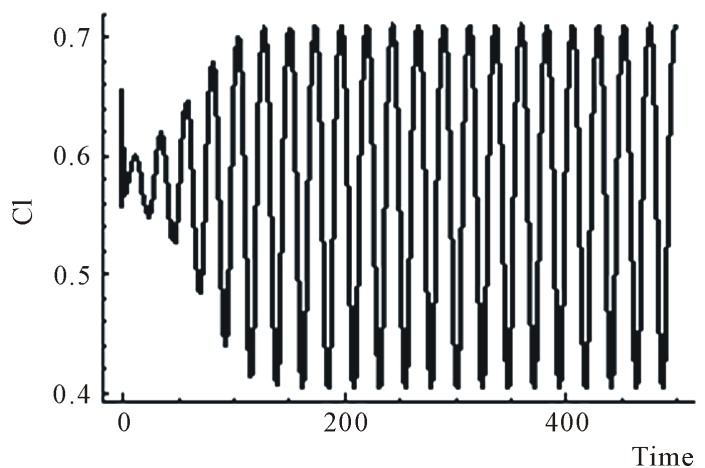

(b)

Figure 8. Results of the transonic buffet of NACA0012 airfoil for the real time $d$ treal = 0.01T. (a) Pressure contours and streamlines of four different times in a period; (b) Lift coefficient response of the airfoil.
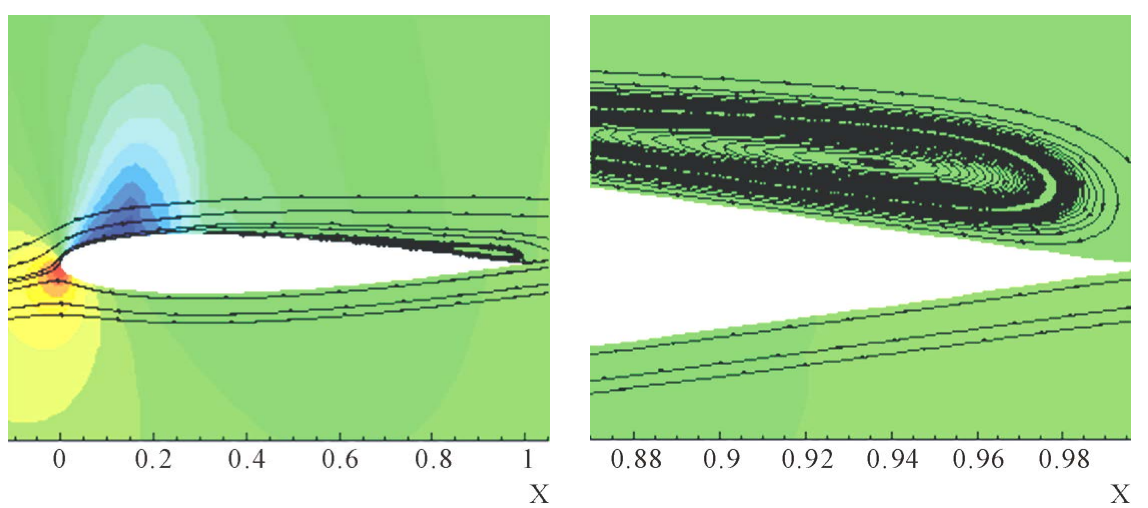

Figure 9. Pressure distribution and streamlines of the time mean flow for NACA0012. 
differences are observed between the time-mean flow and unstable steady flow. For the time-mean flow, the shock wave disappears and the distribution of pressure coefficients becomes smooth. However, for the unstable steady solution, a shock wave remains at $20 \%$ after the leading edge of the airfoil. Furthermore, the values of the convergent coefficient of both flows are different. $\mathrm{Cl}=0.557$ for the time-mean flow, whereas $\mathrm{Cl}=0.578$ for the unstable steady flow. This result is consistent with our preceding examples in the paper. We still perform calculations with different sizes of the real time step and depict the minimum and maximum lift coefficient amplitudes in Figure 11. In this figure, the $y$-axis denotes the amplitudes of the lift coefficient and the $x$-axis denotes
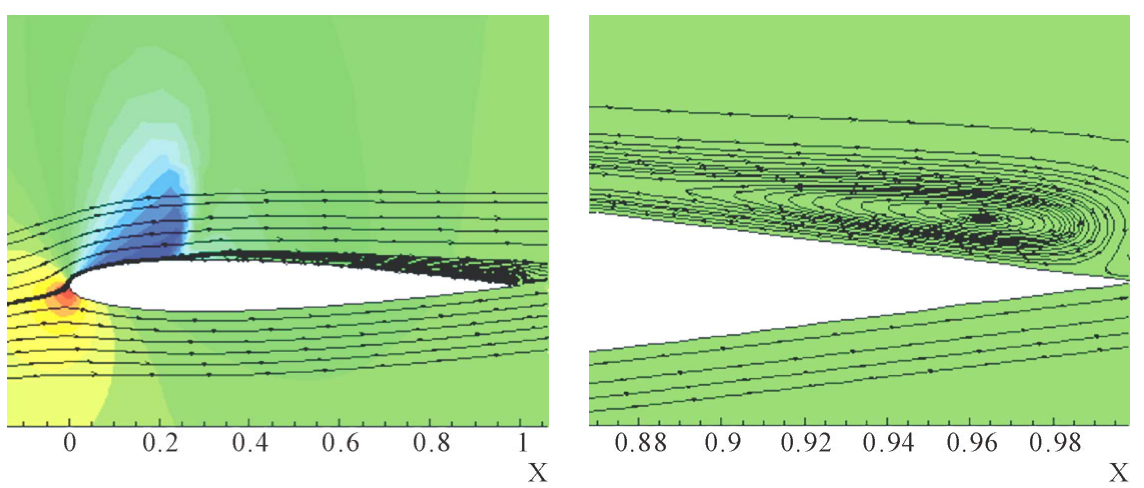

(a)

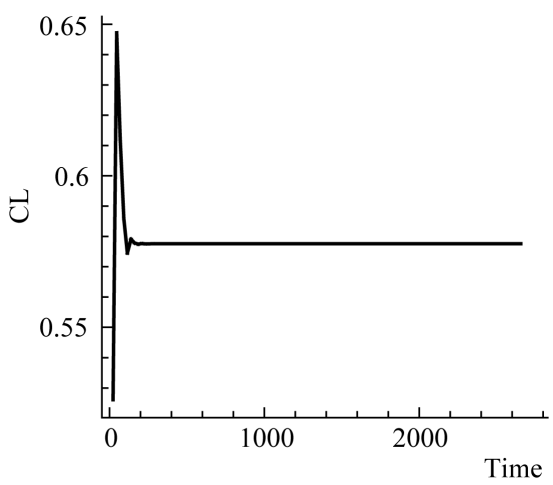

(b)

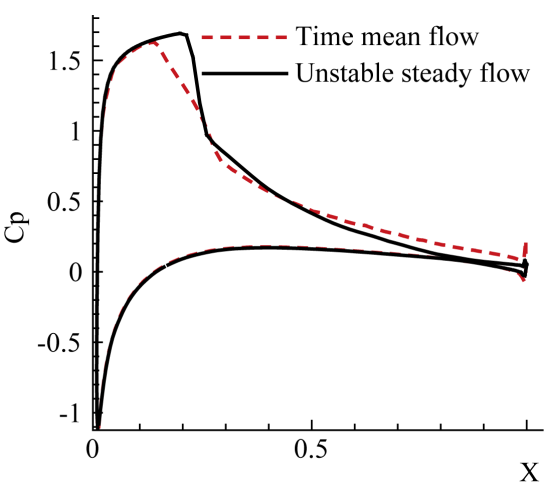

(c)

Figure 10. Results of the transonic buffet of NACA0012 airfoil for the real time dtreal $=T$. (a) Pressure contours and streamlines of the flow field; (b) Lift coefficient response of the airfoil; (c) Comparison of pressure coefficients of time mean flow and unstable steady flow.

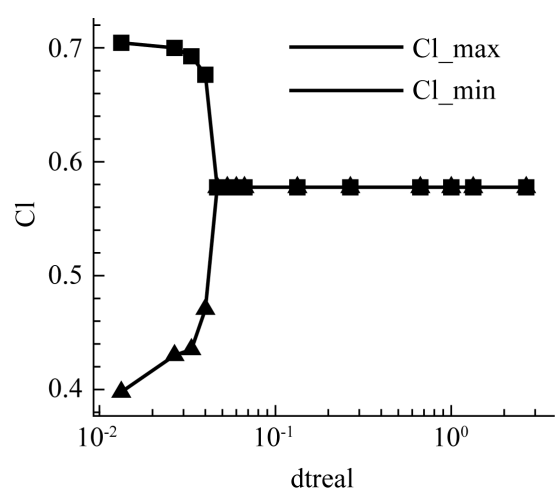

Figure 11. Minimum and maximum amplitudes of the lift coefficient corresponding to different sizes of the real time step. 
the multiple of the shedding vortex period $T$, where $C l \_$min denotes the minimum amplitude of the lift coefficient and $\mathrm{Cl}$-max denotes the maximum amplitude of the lift coefficient. The critical real time step is $d t r e a l=$ $5 \% T$. When the real time step is larger than the critical step, the flow field will tend to remain stable and have the same size of the lift coefficient.

\section{Conclusion}

This paper proposes a fast, effective, and accurate way of capturing the unstable steady solution. By enlarging the real time step size, the unstable frequency modes of the unsteady flow field are filtered out because of the damping in the real time level. When the numerical iteration converges, the time derivatives of unsteady term dismiss automatically and the stable solution suited for steady governing equations are obtained. The method is validated by two unsteady flow cases including the laminar flow past a circular cylinder and a transonic buffet of NACA0012 airfoil. The critical real time step of acquiring the steady flow field is related to the instability strength of the flow field. A stronger instability characteristic corresponds to the need for a larger a critical real step size. The unstable steady solution can be obtained by this method. Thus, we can recalculate the unsteady flow field. Moreover, the detailed process from the linear state to the unsteady nonlinear saturated state can be captured, thus providing the foundation for the modeling and analysis of unstable flows.

\section{References}

[1] Dowell, E.H. and Hall, K.C. (2001) Modeling of Fluid-Structure Interaction. Annual Reviews of Fluid Mechanics, 33, 445-490. http://dx.doi.org/10.1146/annurev.fluid.33.1.445

[2] Lucia, D.J., Beran, P.S. and Silva, W.A. (2004) Reduced-Order Modeling: New Approaches for Computational Physics. Progress in Aerospace Sciences, 40, 51-117. http://dx.doi.org/10.1016/j.paerosci.2003.12.001

[3] Silva, W. (2005) Identification of Nonlinear Aeroelastic Systems Based on the Volterra Theory: Progress and Opportunity. Nonlinear Dynamics, 39, 25-62. http://dx.doi.org/10.1007/s11071-005-1907-z

[4] Amsallem, D. and Farhat, C. (2008) Interpolation Method for Adapting Reduced-Order Models and Application to Aeroelasticity. AIAA Journal, 46, 1803-1813. http://dx.doi.org/10.2514/1.35374

[5] Marzocca, P., Silva, W.A. and Librescu, L. (2004) Nonlinear Open/Closed-Loop Aeroelastic Analysis of Airfoils via Volterra Series. AIAA Journal, 42, 673-686. http://dx.doi.org/10.2514/1.9552

[6] Zhang, W.W., Ye, Z.Y. and Zhang, C.A. (2009) Aeroservoelastic Analysis for Transonic Missile Based on Computational Fluid Dynamics. Journal of Aircraft, 46, 2178-2183. http://dx.doi.org/10.2514/1.45249

[7] Zhang, W.W. and Ye, Z.Y. (2010) Effect of Control Surface on Airfoil Flutter in Transonic Flow. Acta Astronautica, 66, 999-1007. http://dx.doi.org/10.1016/j.actaastro.2009.09.016

[8] Zhang, W.W., Wang, B.B. and Ye, Z.Y. (2012) Efficient Method for Limit Cycle Flutter Analysis by Nonlinear Aerodynamic Reduced-Order Models. AIAA Journal, 50, 1019-1028. http://dx.doi.org/10.2514/1.J050581

[9] Williamson, C.H.K. and Govardhan, R. (2004) Vortex-Induced Vibrations. Annual Review of Fluid Mechanics, 36, 431-455. http://dx.doi.org/10.1146/annurev.fluid.36.050802.122128

[10] Bearman, P.W. (2011) Circular Cylinder Wakes and Vortex-Induced Vibrations. Journal of Fluids and Structures, 27, 648-658. http://dx.doi.org/10.1016/j.jfluidstructs.2011.03.021

[11] Sengupta, T.K., Bhole, A. and Sreejith, N.A. (2013) Direct Numerical Simulation of 2D Transonic Flows around Airfoils. Computers \& Fluids, 88, 19-37. http://dx.doi.org/10.1016/j.compfluid.2013.08.007

[12] Alshabu, A. and Olivier, H. (2008) Unsteady Wave Phenomena on a Supercritical Airfoil. AIAA Journal, 46, 20662073. http://dx.doi.org/10.2514/1.35516

[13] Iovnovich, M. and Raveh, D.E. (2012) Reynolds-Averaged Navier-Stokes Study of the Shock-Buffet Instability Mechanism. AIAA Journal, 50, 880-890. http://dx.doi.org/10.2514/1.J051329

[14] Crouch, J.D., Garbaruk, A. and Magidov, D. (2007) Predicting the Onset of Flow Unsteadiness Based on Global Instability. Journal of Computational Physics, 224, 924-940. http://dx.doi.org/10.1016/j.jcp.2006.10.035

[15] Crouch, J.D., Garbaruk, A., Magidov, D. and Travin, A. (2009) Origin of Transonic Buffet on Aerofoils. Journal of Fluid Mechanics, 628, 357-369. http://dx.doi.org/10.1017/S0022112009006673

[16] Woodgate, M.A. and Badcock, K.J. (2007) Fast Prediction of Transonic Aeroelastic Stability and Limit Cycles. AIAA Journal, 45, 1370-1381. http://dx.doi.org/10.2514/1.25604

[17] Illingworth, S.J., Morgans, A.S. and Rowley, C.W. (2012) Feedback Control of Cavity Flow Oscillations Using Simple Linear Models. Journal of Fluid Mechanics, 709, 223-248. http://dx.doi.org/10.1017/jfm.2012.330 
[18] Ahuja, S. and Rowley, C.W. (2010) Feedback Control of Unstable Steady States of Flow past a Flat Plate Using Reduced-Order Estimators. Journal of Fluid Mechanics, 645, 447-478. http://dx.doi.org/10.1017/S0022112009992655

[19] Barkley, D. (2006) Linear Analysis of the Cylinder Wake Mean Flow. Europhysics Letters, 75, 750-756. http://dx.doi.org/10.1209/epl/i2006-10168-7

[20] Jessie, W., Simone, C. and Angelo, I. (2009) Feedback Control by Low-Order Modeling of the Laminar Flow past a Bluff Body. Journal of Fluid Mechanics, 634, 405-418. http://dx.doi.org/10.1017/S0022112009990590

[21] He, N., Tourlidakis, A. and Elder, R.L. (2007) Comparisons of Steady and Time-Averaged Unsteady Flow Predictions for Impeller-Diffuser Interactions in a Centrifugal Compressor Stage. Proceedings of GT2007 ASME Turbo Expo 2007: Power for Land, Sea and Air, Montreal, 14-17 May 2007. http://dx.doi.org/10.1115/gt2007-27985

[22] Chen, Z.L. and Merkle, C.L. (2011) Contrast between Steady and Time-Averaged Unsteady Combustion Simulations. Computers \& Fluids, 44, 328-338. http://dx.doi.org/10.1016/j.compfluid.2011.01.032

[23] Mittal, S. (2008) Global Linear Stability Analysis of Time-Averaged Flows. International Journal for Numerical Methods in Fluids, 58, 111-118. http://dx.doi.org/10.1002/fld.1714

[24] Langhorne, P.J., Dowling, A.P. and Hooper N. (1990) Practical Active Control System for Combustion Oscillations. Journal of Propulsion and Power, 6, 324-333. http://dx.doi.org/10.2514/3.25437

[25] Tieron, J.E. and Doyle, J.C. (1992) Multi Mode Active Stabilization of a Rijke Tube. Proceedings of the ASME Winter Annual Meeting, 38, 65-68.

[26] Roe, P.L. (1981) Approximate Riemann Solvers, Parameter Vectors, and Difference Schemes. Journal of Computational Physics, 43, 357-372. http://dx.doi.org/10.1016/0021-9991(81)90128-5

[27] Jameson, A. (1991) Time Dependent Calculations Using Multigrid, with Applications to Unsteady Flows past Airfoils and Wings. Proceedings of the AIAA 10th Computational Fluid Dynamics Conference, Honolulu, 24-26 June 1991. 